\title{
Management of acute ischemic stroke due to tandem occlusion: should endovascular recanalization of the extracranial or intracranial occlusive lesion be done first?
}

\author{
Leonardo Rangel-Castilla, MD, ${ }^{1,5,6}$ Gary B. Rajah, MD, ${ }^{6}$ Hakeem J. Shakir, MD, ${ }^{1,5}$ \\ Hussain Shallwani, MD, ${ }^{1,5}$ Sirin Gandhi, MD,, 4,5 Jason M. Davies, MD, PhD, 1,2,5 \\ Kenneth V. Snyder, MD, PhD, ${ }^{1,4,5,7}$ Elad I. Levy, MD, MBA, ${ }^{1,3,5,7}$ and Adnan H. Siddiqui, MD, PhD, , 1,3,5,7, \\ Departments of ${ }^{1}$ Neurosurgery, ${ }^{2}$ Biomedical Informatics, ${ }^{3}$ Radiology, and ${ }^{4}$ Neurology, Jacobs School of Medicine and Biomedical \\ Sciences, and ${ }^{7}$ Toshiba Stroke and Vascular Research Center, University at Buffalo, State University of New York; ${ }^{5}$ Department \\ of Neurosurgery, Gates Vascular Institute at Kaleida Health; ${ }^{8} \mathrm{Jacobs}$ Institute, Buffalo, New York; and ${ }^{6}$ Department of \\ Neurosurgery, Wayne State School of Medicine, Wayne State University, Detroit, Michigan
}

OBJECTIVE Acute tandem occlusions of the cervical internal carotid artery and an intracranial large vessel present treatment challenges. Controversy exists regarding which lesion should be addressed first. The authors sought to evaluate the endovascular approach for revascularization of these lesions at Gates Vascular Institute.

METHODS The authors performed a retrospective review of a prospectively maintained, single-institution database. They analyzed demographic, procedural, radiological, and clinical outcome data for patients who underwent endovascular treatment for tandem occlusions. A modified Rankin Scale (mRS) score $\leq 2$ was defined as a favorable clinical outcome.

RESULTS Forty-five patients were identified for inclusion in the study. The average age of these patients was 64 years; the mean National Institutes of Health Stroke Scale score at presentation was 14.4. Fifteen patients received intravenous thrombolysis before undergoing endovascular treatment. Thirty-seven (82\%) of the 45 proximal cervical internal carotid artery occlusions were atherothrombotic in nature. Thirty-eight patients underwent a proximal-to-distal approach with carotid artery stenting first, followed by intracranial thrombectomy, whereas 7 patients underwent a distal-to-proximal approach (that is, intracranial thrombectomy was performed first). Thirty-seven (82\%) procedures were completed with local anesthesia. For intracranial thrombectomy procedures, aspiration alone was used in 15 cases, stent retrieval alone was used in 5, and a combination of aspiration and stent-retriever thrombectomy was used in the remaining 25 . The average time to revascularization was 81 minutes. Successful recanalization (thrombolysis in cerebral infarction Grade $2 \mathrm{~b} / 3$ ) was achieved in 39 (87\%) patients. Mean National Institutes of Health Stroke Scale scores were 9.3 immediately postprocedure $(p<0.05)(n=31), 5.1$ at discharge $(p<0.05)(n=31)$, and 3.6 at 3 months $(p<0.05)(n=30)$. There were 5 in-hospital deaths (11\%); and 2 patients (4.4\%) had symptomatic intracranial hemorrhage within 24 hours postprocedure. Favorable outcomes (mRS score $\leq 2)$ were achieved at 3 months in $22(73.3 \%)$ of 30 patients available for follow-up, with an mRS score of 3 for 7 of $30(23 \%)$ patients.

CONCLUSIONS Tandem occlusions present treatment challenges, but high recanalization rates were possible in the present series using acute carotid artery stenting and mechanical thrombectomy concurrently. Proximal-to-distal and aspiration approaches were most commonly used because they were safe, efficacious, and feasible. Further study in

ABBREVIATIONS CCA = common carotid artery; ICA = internal carotid artery; ICH = intracranial hemorrhage; IV = intravenous; $M C A=$ middle cerebral artery; $m R S=$ modified Rankin Scale; NIHSS = National Institutes of Health Stroke Scale; $\mathrm{TICl}=$ thrombolysis in cerebral infarction; tPA = tissue plasminogen activator.

SUBMITTED November 29, 2016. ACCEPTED January 10, 2017.

INCLUDE WHEN CITING DOI: 10.3171/2017.1.FOCUS16500. 
the setting of a randomized controlled trial is needed to determine the best sequence for the treatment approach and the best technology for tandem occlusion.

https://thejns.org/doi/abs/10.3171/2017.1.FOCUS16500

KEY WORDS acute ischemic stroke; carotid artery occlusion; tandem occlusion; carotid artery stenting; mechanical thrombectomy; aspiration

A CUTE tandem occlusion involving the cervical internal carotid artery (ICA) with concomitant intracranial thromboembolism to the intracranial ICA terminus or middle cerebral artery (MCA) is estimated to occur in $15 \%$ of all cases of large-vessel acute ischemic stroke. ${ }^{17}$ These lesions are challenging to manage endovascularly and respond poorly to intravenous (IV) tissue plasminogen activator (tPA). ${ }^{2}$ Tandem lesions typically have a worse prognosis than an intracranial lesion alone and are associated with higher rates of symptomatic intracranial hemorrhage $(\mathrm{ICH})$, with 1 small series reporting a $22 \%$ rate of symptomatic $\mathrm{ICH}$ and a $39 \%$ rate of 90 -day mortality. ${ }^{3}$

There is controversy regarding which lesion should be treated first (extracranial vs intracranial). Different endovascular approaches have been proposed, including mechanical thrombectomy without attempted treatment of the cervical ICA, angioplasty and stenting of the cervical lesion first, followed by intracranial thrombectomy, or treatment of the intracranial occlusion with mechanical thrombectomy first, followed by treatment of the cervical ICA occlusion. Moreover, controversy regarding the optimal treatment approach has led some authors to speculate on whether acute stenting and dual antiplatelet therapy are appropriate in this setting. ${ }^{17}$ Here we describe our series on the management of tandem occlusion in the setting of acute ischemic stroke. We sought to determine how our outcomes and complications compare with other limited published experiences in this patient group. Furthermore, we assessed various intracranial mechanical thrombectomy techniques for differences in procedural time and outcomes.

\section{Methods}

\section{Patients, Study Design, and Setting}

After receiving institutional review board approval, we retrospectively reviewed a prospectively maintained endovascular database at a single institution. Consecutive cases of emergent endovascular management of acute tandem occlusion were accrued between January 2012 and June 2015. A review of radiological and angiographic images, including from digital subtraction angiography, CT angiography and perfusion imaging, and MRI, was used to identify the patients in these cases. Only those with radiological evidence of tandem occlusion were included in the analysis.

Baseline demographic data and clinical characteristics (e.g., age, sex, functional status, medical history, National Institutes of Health Stroke Scale [NIHSS] score, and radiological information) were obtained by a review of electronic medical records. As reported previously, ${ }^{10}$ we routinely evaluate patients who present with stroke- like symptoms using noncontrast head CT imaging, head and neck CT angiography (including 4D [temporally resolved] CT angiography), and CT perfusion imaging. Patients with unfavorable perfusion profiles routinely are not deemed candidates for endovascular revascularization.

\section{Endovascular Procedure}

Procedures were performed under conscious sedation and local anesthesia except for patients who did not cooperate due to severe aphasia. Some patients received tPA in the emergency department according to the American Heart Association/American Stroke Association guidelines. ${ }^{4}$ The endovascular neurosurgeon reviewed the CT angiogram (Figs. 1A-C and 2A), 4D CT angiogram, and $\mathrm{CT}$ perfusion images. A tandem occlusion was diagnosed based on simultaneous presence of a cervical and intracranial occlusion both on conventional CT angiography as well as on 4D CT angiography.

CT perfusion imaging was used to exclude patients with a large established core ( $>50 \%$ of at-risk territory). These patients received a loading dose of dual antiplatelet medications (aspirin $650 \mathrm{mg}$ and clopidogrel $600 \mathrm{mg}$ or ticagrelor $180 \mathrm{mg}$ ) prior to the acute endovascular intervention. Systemic heparinization is typically not used at our institution during intracranial thrombectomy. However, when cervical occlusion was confirmed during angiography, a systemic loading dose of heparin was delivered to achieve an activated coagulation time of $\geq 250$ seconds. Diagnostic angiography was performed to confirm the occlusion pattern and collateral supply (Figs. 1D and 2B).

In general, a 90-cm, 6-Fr guiding catheter or 9-Fr balloon guiding catheter, including the 9-Fr Mo.Ma dual balloon guide catheter (Medtronic), was placed at the distal common carotid artery (CCA) ipsilateral to the occluded ICA (Fig. 3A-E). When positioning the Mo.Ma guide catheter, the distal balloon was positioned in the external carotid artery while the proximal guide was positioned in the CCA. The cervical ICA was explored with a 0.014inch microguidewire and a microcatheter after inflation of the proximal balloon. During this step, the endovascular neurosurgeon evaluated the status of the cervical ICA. The presence of any resistance while passing the microcatheter or microguidewire indicated the presence of an occlusive cervical ICA stenosis.

After distal access was obtained (petrocavernous ICA or farther), angiography was performed through the microcatheter to assess ICA status. An exchange 014 wire was then positioned in the petrocavernous ICA, and angioplasty and stenting of the cervical ICA was performed using a closed-cell stent sized to the CCA (Figs. 1E and $2 \mathrm{C})$. Frequently, intravascular ultrasound imaging was used to confirm patency and to identify luminal thrombus, 

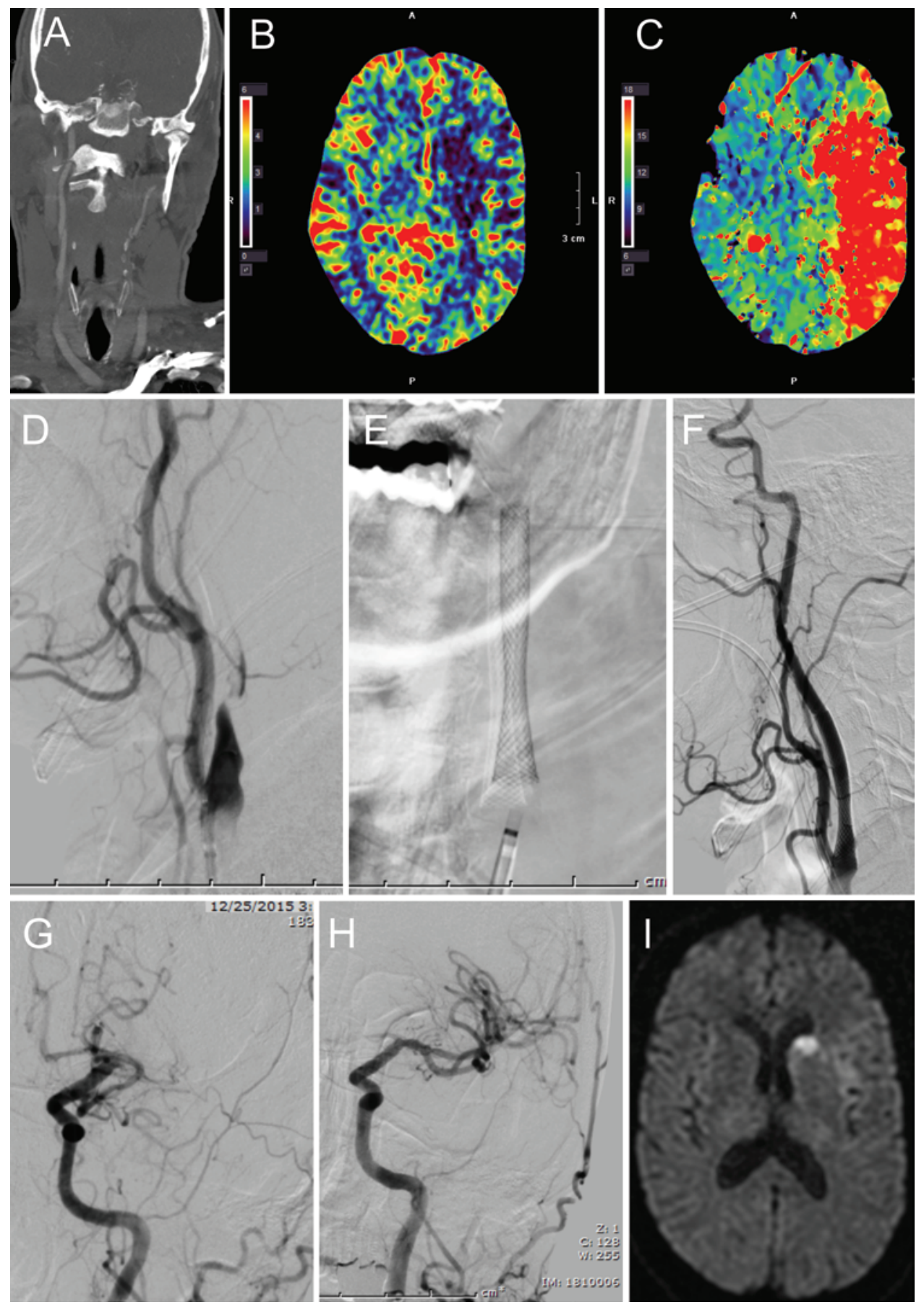

FIG. 1. Left cervical ICA and MCA tandem occlusions. This 59-year-old man presented with acute ischemic stroke and an NIHSS score of 18. A: CT angiogram demonstrating complete occlusion of the left cervical ICA. B and C: CT perfusion images demonstrating preserved volume with increased time-to-peak in the left cerebral hemisphere. D: Left CCA angiogram demonstrating complete occlusion of the cervical ICA (flame shape). E: Cervical radiograph showing the tip of the guide catheter and the cervical stent. F: Immediate poststenting left cervical ICA angiogram showing successful revascularization of the cervical and intracranial ICA. G: Left ICA injection angiogram, cranial projection, demonstrating complete occlusion of the left MCA. H: Left ICA injection angiogram, cranial projection, demonstrating complete revascularization of the left MCA after 1-pass aspiration for intracranial thrombectomy. I: 24-hour postprocedure diffusion-weighted MR image showing a small caudate infarction. At 24 hours, the patient's NIHSS score was 1.

which was addressed with direct aspiration or additional stenting prior to restoration of anterograde flow. In some cases, at the discretion of the endovascular neurosurgeon, a distal embolic protection device was used instead of a proximal dual balloon Mo.Ma guide. This was most likely if there was very high-grade stenosis, rather than true cervical ICA occlusion (pseudo-occlusion). It was in this subset, after the cervical lesion was crossed, that intracranial 


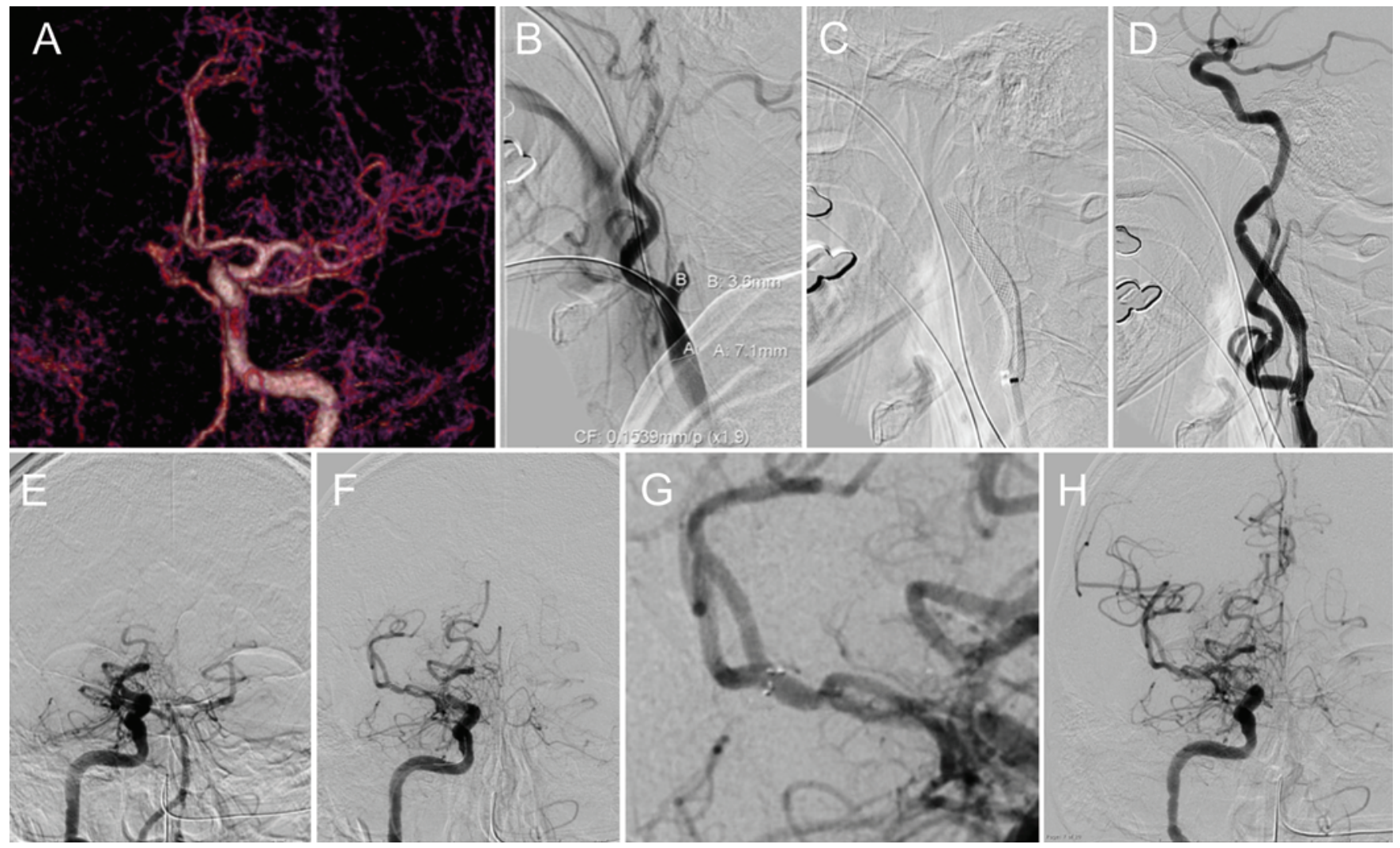

FIG. 2. Right cervical ICA and MCA tandem occlusions. This 65 -year-old man presented with acute ischemic stroke and an NIHSS score of 20. A: CT angiogram demonstrating complete occlusion of the right cervical ICA. B: Right CCA angiogram, cervical view, showing complete occlusion of the right ICA. C: Lateral radiograph showing the guide catheter and a deployed closed-cell carotid artery stent. D: Poststenting right CCA angiogram demonstrating successful revascularization of the right ICA. E: Right CCA angiogram, cranial view, showing complete occlusion of the right MCA. F: Right ICA angiogram, cranial view, showing the stent retriever deployed at the MCA with revascularization of the vessel. G: Magnification view of the right MCA with the stent retriever. H: Right ICA angiogram, cranial view, obtained after mechanical thrombectomy, showing complete revascularization of the right ICA and MCA.

thrombectomy was performed first and cervical stenting under distal protection was performed after intracranial revascularization, if needed.

Carotid artery angiography was then performed to assess ICA recanalization and to determine the precise location and extent of the distal occlusion (Figs. 1F and 2D). At this point, if a single balloon guide was used, it was deflated and simply advanced through the newly stented ICA into the distal ICA beyond the stent. On the other hand, if the Mo.Ma dual balloon guide was used, then both balloons were deflated and the guide was replaced using a standard 035 exchange wire with a 6-Fr sheath or an 8- or 9-Fr single balloon guide catheter into the distal cervical ICA beyond the stent.

Over the course of this analysis, several new techniques were developed and incorporated into practice as they became available. Options included a direct aspiration firstpass technique (ADAPT) ${ }^{15}$ and stent-retriever thrombectomy ${ }^{9}$ (Figs. $1 \mathrm{G}$ and $2 \mathrm{E}-\mathrm{G}$ ). The decision to use aspiration versus stent-retriever thrombectomy was at the discretion of the treating endovascular neurosurgeon.

A final angiogram was performed to assess revascularization of the ICA and its branches (Figs. $1 \mathrm{H}$ and $2 \mathrm{H}$ ).
Immediate postprocedural $\mathrm{CT}$ imaging was performed to detect hemorrhagic complications or to assess the need for a further procedure. At 24 hours postprocedure, MRI was performed to evaluate the extension of the acute stroke (Fig. 1I). Patients were maintained on dual antiplatelet medications on a daily basis ( $75 \mathrm{mg}$ of clopidogrel for 3 months and $325 \mathrm{mg}$ of aspirin for life).

\section{Data Sources/Measurement}

We evaluated radiological and clinical outcomes. The degree of recanalization was graded according to the thrombolysis in cerebral infarction (TICI) grade on the last angiographic run (successful recanalization, TICI Grade $2 b / 3$ ). Postprocedural ICH was determined by a review of head CT studies performed within the 30-day postprocedural time frame. Symptomatic hemorrhage was classified based on an increase in NIHSS score by 4 points or death related to ICH. Any adverse clinical events attributable to the procedure were noted. Clinical outcome was assessed according to the NIHSS score at the time of hospital discharge and last clinical follow-up. Modified Rankin Scale (mRS) scores were assessed at 90 days. 

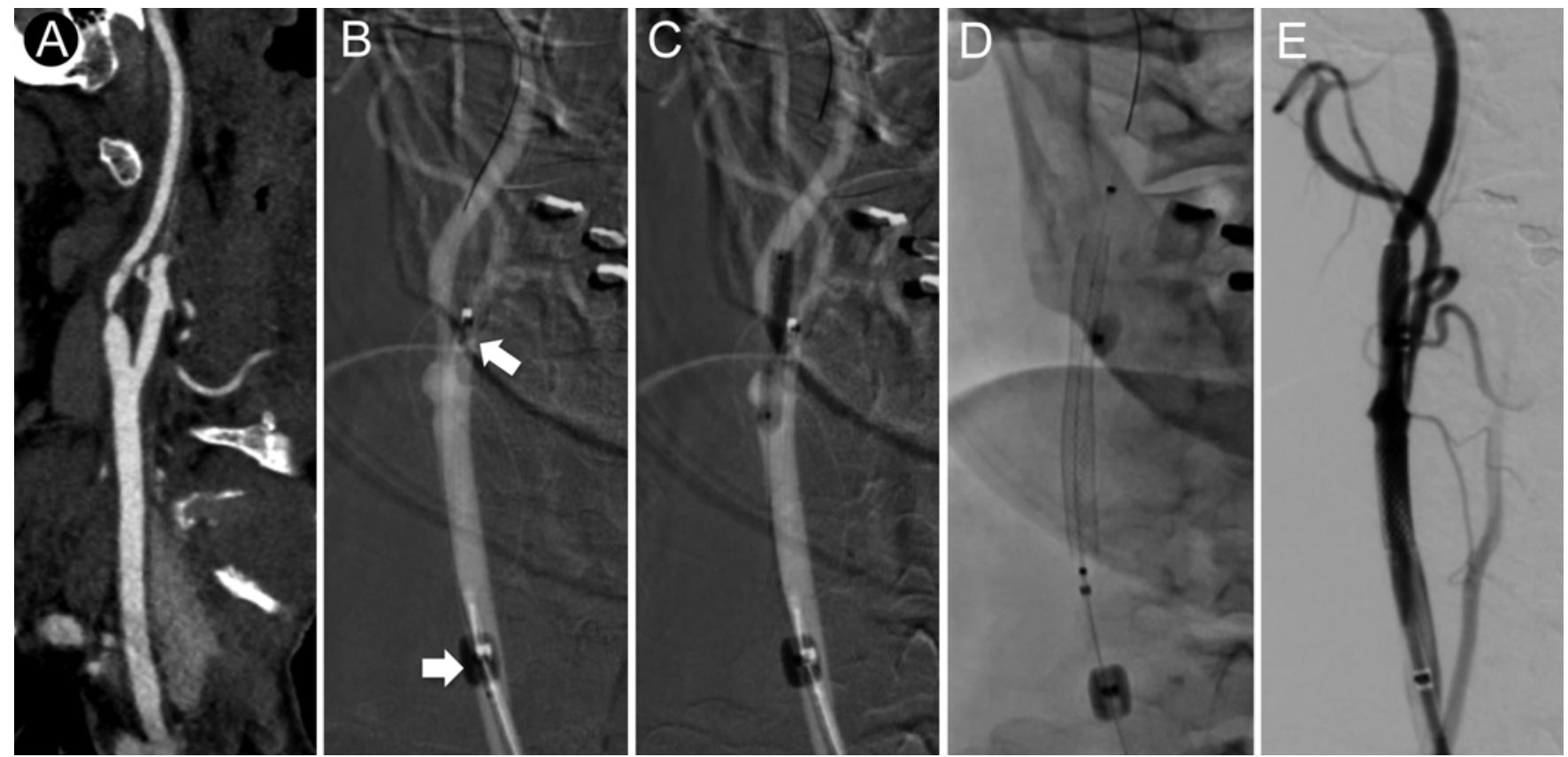

FIG. 3. Extracranial carotid artery stenting under flow arrest. A: CT angiogram demonstrating severe stenosis at the extracranial ICA in a patient presenting after a transient ischemic attack. B: Anteroposterior view of the right ICA with a dual balloon guide catheter (Mo.Ma guide catheter) used for flow arrest. Arrows indicate balloons at the common and external carotid arteries. C: Prestenting balloon angioplasty. D: Anteroposterior radiograph showing the stent being deployed. E: Poststenting anteroposterior view of the right ICA angriogram demonstrating significant improvement of the severe stenosis.

\section{Statistical Analysis}

Two-tailed paired t-tests were used for statistical analysis of NIHSS scores pre- and postintervention. ANOVA was used to assess for significance in NIHSS scores between the stent retriever alone, aspiration alone, and combined groups. Excel (version 14.2.0; Microsoft) and SPSS (IBM) were used for statistical analysis.

\section{Results}

\section{Demographic Data and Clinical Characteristics}

We identified 45 patients who presented with acute tandem occlusion and underwent endovascular therapy during the study period. Baseline demographic data and clinical information are presented in Table 1 . The average age of patients was 64 years, and the mean NIHSS score at presentation was 14.4 (median 15.5, SD 5.6). The TICI grade at initial evaluation was 0 in all patients. The tandem occlusion occurred on the left side in 24 patients (53\%). Fifteen patients (33\%) received IV tPA before undergoing endovascular intervention. Thirty-seven of 45 proximal cervical ICA occlusions (82\%) were atherothrombotic versus dissection. The locations of distal occlusion sites are also provided in Table 1.

\section{Endovascular Technique}

Thirty-eight patients $(84.5 \%)$ underwent a proximalto-distal approach (anterograde carotid artery stenting followed by intracranial thrombectomy) and 7 (15.5\%) underwent a distal-to-proximal approach (intracranial thrombectomy followed by carotid artery stenting). Nine patients
(20\%) required $>1$ carotid artery stent. Numbers and types of stents used included 33 WALLSTENTs (Boston Scientific), 1 Enterprise (Codman), 5 Xpert (Abbott Vascular), 4 XACT (Abbott Vascular), 1 Wingspan (Stryker), and 1 Pipeline (Medtronic). Single or dual balloon guide catheters were used in 25 patients (55.5\%). The use of a balloon guide catheter was at the discretion of the operating endovascular neurosurgeon.

For intracranial thrombectomy procedures, aspiration alone was successfully used for 15 of the 45 cases (33\%), and stent-retriever thrombectomy alone was used in 5 (11\%). The aspiration-alone group required 1 pass in 12 of the 15 cases (80\%). A combination of aspiration and stent retrieval was used for intracranial thrombectomy in the remaining 25 cases. Of the 30 cases using stent retrievers, 22,7 , and 1 cases required 1,2 , and $>2$ passes, respectively. No statistical differences in procedural time or complications were noted between the aspiration-alone versus stent retriever-alone groups. The average time from groin puncture to revascularization was 81 minutes.

\section{Outcome Data and Analysis}

Successful recanalization (TICI Grade 2b/3) was achieved in $39(87 \%)$ of 45 patients. Compared with the presentation NIHSS scores, the postprocedure, discharge, and 3-month NIHSS scores were $9.3(\mathrm{p}<0.05)(\mathrm{n}=31)$, $5.1(\mathrm{p}<0.05)(\mathrm{n}=31)$, and $3.6(\mathrm{p}<0.05)(\mathrm{n}=30)$. There were 5 deaths $(11 \%)$ during hospitalization, of which 2 were related to large infarcts and malignant cerebral edema, 1 to a symptomatic ICH, and the other 2 to cardiac comorbidities. At 24 hours, 8 patients (18\%) had evidence 
TABLE 1. Baseline demographic data and clinical characteristics of 45 patients with tandem occlusion

\begin{tabular}{lc}
\hline \multicolumn{1}{c}{ Characteristic } & Value (\%) \\
\hline Average age, yrs & 64 \\
\hline Female sex, no. of pts & $18(40)$ \\
\hline Smoking, no. of pts & $15(33)$ \\
\hline Hyperlipidemia/cholesterolemia, no. of pts & $24(53)$ \\
\hline Hypertension, no. of pts & $34(75)$ \\
\hline Diabetes, no. of pts & $10(22)$ \\
\hline Mean NIHSS score at presentation & 14.4 \\
\hline Mean time from symptom onset to arrival, mins & 139 \\
\hline Proximal stenosis vs occlusion, no. of pts & 7 vs 38 (mean \\
& stenosis $86 \%)$ \\
\hline Type of lesion proximally, no. of pts & $37(82)$ \\
\hline Thrombotic & 7 \\
\hline Distal clot location, no. of pts & 10 \\
\hline ICA-T & 1 \\
\hline ICA-L & 20 \\
\hline ICA-iso & 7 \\
\hline $\mathrm{M}_{1}$ & \\
\hline $\mathrm{M}_{2}$ &
\end{tabular}

ICA-iso = only the terminus; ICA-L = ICA terminus with $M_{1}$ segment involvement; ICA-T = ICA terminus with involvement of the $M_{1}$ or $A_{1}$ segment; $p t s=$ patients.

of ICH on CT imaging; only 2 of them were symptomatic (4.4\%). Procedural complications included 1 iatrogenic arterial dissection that required another cervical ICA stent. In the other case of dissection, an Xpert stent in the proximal ICA was moved by the stent-retriever device slightly proximal into the common ICA during thrombectomy, requiring an additional stent at the proximal ICA.

Vasospasm occurred in 3 patients; verapamil infusion was required in 1 patient only. There were 2 cases of immediate stent thrombosis in the ICA; both were opened with an IV loading dose of eptifibatide (2b-3a antagonist). Both patients had mRS scores of 0 . There was 1 case of intraprocedural contrast extravasation, and this patient had an mRS score of 2 at 3 months. At 3-months' follow-up, favorable outcomes $(\mathrm{mRS} \geq 2)$ were achieved by 22 of the 45 patients in our series (49\%), and 7 patients $(15 \%)$ had an mRS score of 3 (by that time, 11 patients were lost to follow-up and the remaining 5 patients had died in the hospital, as mentioned previously). The mRS scores by intracranial occlusion location are listed in Table 2; of note, mRS scores of 0 were associated with $\mathrm{M}_{1}$ occlusion more often than ICA terminus or long segment occlusions. Procedural times, characteristics, and outcomes are provided in Table 2 . The difference in time to recanalization between groups was not statistically significant $(95 \%$ CI $79 \pm 11.7$ minutes for the stenting-first group vs $91 \pm 27.4$ minutes for the thrombectomy-first group).

\section{Discussion}

Acute ischemic stroke related to tandem occlusion of the cervical ICA and an intracranial large vessel (e.g., the ICA terminus or MCA) presents a challenge to the endo-
TABLE 2. Procedural times, characteristics, and outcomes

\begin{tabular}{|c|c|}
\hline Treatment Characteristic & $\begin{array}{c}\text { Outcome, } \\
\text { No. }(\%)\end{array}$ \\
\hline Avg recanalization time, mins & 81 \\
\hline $\mathrm{TICl} 2 \mathrm{~b} / 3$ recanalization, no. of pts & $39(87)$ \\
\hline Primary carotid stent placement & $38(84.5)$ \\
\hline \multicolumn{2}{|l|}{ Avg time to revascularization, mins } \\
\hline w/ stent retriever alone & 60 \\
\hline w/ aspiration alone & 60 \\
\hline w/ aspiration + stent retriever & 94 \\
\hline \multicolumn{2}{|l|}{ Stent placement, avg time to revascularization, mins } \\
\hline Primary stent (proximal-to-distal approach) & 79 \\
\hline Secondary stent (distal-to-proximal approach) & 91 \\
\hline \multicolumn{2}{|l|}{ NIHSS score } \\
\hline Postprocedure & $9.3(n=31)$ \\
\hline Discharge & $5.1(n=31)$ \\
\hline 3 mos postprocedure & $3.6(n=30)$ \\
\hline$m R S$ score $\leq 2$ at 90 days & 22 of $45(49)^{\prime}$ \\
\hline \multicolumn{2}{|l|}{ mRS score by intracranial occlusion location } \\
\hline 0 & 10 \\
\hline$M_{1}$ & 8 \\
\hline ICA-T & 1 \\
\hline ICA-L & 1 \\
\hline 1 & 5 \\
\hline$M_{1}$ & 3 \\
\hline ICA-T & 1 \\
\hline $\mathrm{M}_{2}$ & 1 \\
\hline 2 & 7 \\
\hline ICA-L & 3 \\
\hline ICA-T & 1 \\
\hline$M_{1}$ & 2 \\
\hline $\mathrm{M}_{2}$ & 1 \\
\hline
\end{tabular}

* Only $22(73.3 \%)$ of 30 patients were available for follow-up at that time.

vascular neurosurgeon. Intravenous tPA is not effective and has a very low rate of recanalization in these cases due to the degree of clot burden and hemodynamic compromise, which result in poor drug delivery. ${ }^{5,8}$ The optimal endovascular approach remains controversial. The distalto-proximal method has the advantage of reducing time to recanalization of the intracranial vessel, thus decreasing the duration of ischemia if there are adequate intracranial collaterals, whereas primary stenting improves these collaterals while distal recanalization is performed.

Our approach was unique because the vast majority of primary stenting cases were performed using a proximal flow reversal/flow arrest strategy with the dual balloon Mo.Ma guide catheter. This guide was used in the Embolic Protection with Reverse Flow (EMPiRE) high-risk carotid artery angioplasty and stenting registry and resulted in a total 30-day major adverse event (stroke, myocardial infarction, or death) rate of $4.5 \% .^{1}$ The unique advantage of this system is that the lesion can be crossed with any tool. In most of our cases, we were successful using a 014 
microwire-microcatheter combination. However, if any difficulty was encountered, we rapidly upgraded to a much more robust system, such as the 035 wire Quick-Cross peripheral catheter (Cook Medical) without concern about distal embolization.

Similarly, we were able to proceed with robust angioplasty to the nominal diameter of the distal ICA, followed by stenting based on the nominal diameter of the CCA under flow arrest. Recanalization was confirmed using intravascular ultrasound imaging. In cases of the continued presence of luminal thrombus or plaque anywhere in the cervical carotid artery, we were able to aspirate it or pin it to the wall using an additional stent. Thereafter, anterograde flow was restored and intracranial occlusion was evaluated. The one major downside is that current thrombectomy catheter systems do not have adequate length to allow intracranial delivery through the Mo.Ma guide catheter. Therefore, the Mo.Ma guide catheter has to be exchanged for an appropriate distal cervical balloon or regular guide catheter after cervical revascularization.

In our study, we noted shorter times from groin puncture to final recanalization with primary stenting. However, no statistical differences were found in the ultimate recanalization rates or clinical outcomes. Our rationale is that anterograde flow restoration provides better likelihood of collateral restoration and, at least theoretically, reduces the intraprocedural rate of ongoing distal embolization by addressing the causative thromboembolic cervical plaque first. Furthermore, using proximal flow arrest/reversal facilitates rapid crossing of the cervical occlusive lesion. Finally, delivery of distal cervical guide catheters, including balloon guide catheters, inherently requires crossing the causative ruptured thrombotic plaque. This step alone is likely to further disrupt an already unstable plaque, with resultant distal embolization.

In their recent meta-analysis, Sivan-Hoffmann et al. ${ }^{12}$ reported 11 studies that included a total of 237 patients treated with acute stenting for tandem occlusions between 2010 and 2015. Of these patients, 81\% underwent stent placement followed by stent-retriever thrombectomy. The mean NIHSS score was 17 , with recanalization rates reaching $81 \%$. Pooled estimates showed favorable outcome in $44 \%$ of patients, with a $7 \%$ symptomatic ICH rate. In this meta-analysis, the largest of the studies, by Maurer et al., consisted of 43 patients and reported TICI Grade 2b/3 recanalization in $76 \%$ of patients, $\mathrm{mRS}$ scores $\leq 2$ in $32 \%$ of patients at discharge, and an average time of 103 minutes from first angiographic run to recanalization.

In the current report, we present a case series of 45 patients with acute anterior circulation tandem occlusions primarily treated endovascularly with the primary stenting or proximal-to-distal approach. Our data suggest that carotid artery stenting of the proximal occlusion followed by intracranial mechanical thrombectomy is feasible, safe, and effective. Our study compares favorably with the previously mentioned studies in terms of outcomes and procedural times. Although our sample is underpowered and difficult to assess for statistical benefit (only 7 patients had distal thrombectomy followed by proximal stenting, compared with 38 patients undergoing proximal stenting followed by distal thrombectomy), we found that both approaches were feasible.
Other authors have found that the distal-to-proximal approach (thrombectomy first) had shorter mean angiography times and better outcomes (52\% vs 33\%) compared with the proximal-to-distal approach. ${ }^{6}$ A report by Soize et al., ${ }^{13}$ which evaluated 11 patients with tandem occlusion versus 31 patients with single occlusion, noted that tandem lesions were associated with worse functional outcomes, more symptomatic ICH cases, and higher mortality rate. Those authors concluded that the retrograde (distal-toproximal) approach under conscious sedation may have contributed to these poor outcomes. On reviewing the literature of acute ischemic stroke in the setting of extracranial and intracranial occlusions, Rahme et al. ${ }^{11}$ found that treatment of the coexistent intracranial occlusion usually dictated the final outcome.

Upon examining our series, we found that most cases were in the proximal-to-distal group and performed with local sedation. However, we did not find any significant difference compared with the distal-to-proximal group with respect to time or outcomes. We believe that our results, which reveal better recanalization rates, lower rates of symptomatic hemorrhages, and better clinical outcomes, are reflective of addressing the proximal causative lesion first, thus improving collateralization and reducing further embolization, while allowing better visualization of the distal intracranial lesion.

With respect to angioplasty alone versus acute stenting for extracranial disease, in a large population extracted from the National Inpatient Sample, the mortality rate associated with angioplasty alone was 9\% compared with $3.8 \%$ with acute stenting. ${ }^{16}$ However, no firm conclusions could be drawn; when a panel of internationally recognized interventionists was questioned, generalizations were hard to make and it was concluded that angioplasty versus stenting should be decided on an individual basis. ${ }^{17}$ On the basis of our results, acute stenting seems to be justifiable (although off label) and beneficial to our patient population. The idea behind the benefit of stenting includes the ability to secure an unstable thromboembolic plaque and maintain distal patency.

Stent-retriever therapy in the setting of tandem occlusions was reported as safe and efficacious in a recent metaanalysis. ${ }^{12}$ Steglich-Arnholm et al. ${ }^{14}$ reviewed data from 47 patients with tandem occlusion treated with stent retrievers. They reported $87 \%$ TICI Grade $2 \mathrm{~b} / 3$ recanalization with favorable outcomes at 3 months in $68 \%$ of patients. The rate of symptomatic $\mathrm{ICH}$ was $4 \%$; however, the rate of asymptomatic ICH was $43 \%$, with a $17 \%$ rate of stent thrombosis and $9 \%$ mortality rate. Our study found no significant differences between the aspiration-alone group and the stent retriever-alone group. Therefore, either approach may be an excellent option. It should be noted with caution that dragging a stent retriever through a cervical stent may result in entanglement. Thus it is paramount that cervical guide catheters be advanced through the cervical stent so that the stent retriever is collected beyond the cervical stent.

\section{Use of Antiplatelet Agents and Risk of Hemorrhage}

Our symptomatic hemorrhage rate was $4.4 \%$, significantly less than the $31 \%$ symptomatic $\mathrm{ICH}$ rate reported 
by Heck and Brown, ${ }^{3}$ which they attributed to the administration of a loading dose of abciximab and to increasing age. In our study, eptifibatide was only used twice for acute stent thrombosis; otherwise, heparinization with dual antiplatelet agents was used and was well tolerated with only 2 cases of immediate stent thrombosis, both of which resolved intraprocedurally. Emergent dual antiplatelet loading therapy with heparinization up front may represent a safer alternative than other IV antiplatelet agents when preparing for stenting in tandem lesions. The use of $2 b-3 a$ inhibitors should be reserved for when there is intraprocedural evidence of acute stent thrombosis. In such cases, we simply provided a 1-time IV loading dose without continued infusion, which resulted in resolution of stent occlusion in both cases.

\section{Limitations of the Study}

To our knowledge, this series is one of the larger studies of tandem occlusions from a single center. However, it is retrospective and only includes 45 patients. In addition, the numbers of patients in the proximal-to-distal and distalto-proximal groups were not matched, so it is difficult to assess the comparative efficacy of techniques. However, our data suggest that the proximal-to-distal technique is safe, fast, efficacious, and feasible. Similarly, for intracranial thrombectomy via stent retrieval versus aspiration, our numbers of patients are low, with only 5 patients undergoing stent retrieval alone, making it difficult to obtain statistically significant data. However, aspiration was demonstrated to be safe, fast, and efficacious.

\section{Conclusions}

Tandem occlusion in the setting of acute ischemic stroke remains a therapeutic challenge; however, high recanalization rates were possible using acute carotid artery stenting and intracranial thrombectomy, with acceptable morbidity and mortality rates. The proximal-to-distal or primary stenting approach was most commonly used; it was safe, fast, efficacious, and feasible. Aspiration alone represented a safe and feasible technique for intracranial thrombectomy, without the possibility of the stent retriever getting caught by an extracranial carotid artery stent.

\section{Acknowledgments}

We thank the Wayne State University Research Design and Analysis Unit for statistical analysis. We also thank Paul H. Dressel for preparation of the illustrations and Debra J. Zimmer for editorial assistance (both from University at Buffalo Neurosurgery).

\section{References}

1. Clair DG, Hopkins LN, Mehta M, Kasirajan K, Schermerhorn M, Schönholz C, et al: Neuroprotection during carotid artery stenting using the GORE flow reversal system: 30-day outcomes in the EMPiRE Clinical Study. Catheter Cardiovasc Interv 77:420-429, 2011

2. del Zoppo GJ, Poeck K, Pessin MS, Wolpert SM, Furlan AJ, Ferbert A, et al: Recombinant tissue plasminogen activator in acute thrombotic and embolic stroke. Ann Neurol 32:78-86, 1992

3. Heck DV, Brown MD: Carotid stenting and intracranial thrombectomy for treatment of acute stroke due to tandem occlusions with aggressive antiplatelet therapy may be associated with a high incidence of intracranial hemorrhage. J Neurointerv Surg 7:170-175, 2015

4. Jauch EC, Saver JL, Adams HP Jr, Bruno A, Connors JJ, Demaerschalk BM, et al: Guidelines for the early management of patients with acute ischemic stroke: a guideline for healthcare professionals from the American Heart Association/ American Stroke Association. Stroke 44:870-947, 2013

5. Kim YS, Garami Z, Mikulik R, Molina CA, Alexandrov AV: Early recanalization rates and clinical outcomes in patients with tandem internal carotid artery/middle cerebral artery occlusion and isolated middle cerebral artery occlusion. Stroke 36:869-871, 2005

6. Lockau H, Liebig T, Henning T, Neuschmelting V, Stetefeld $\mathrm{H}$, Kabbasch C, et al: Mechanical thrombectomy in tandem occlusion: procedural considerations and clinical results. Neuroradiology 57:589-598, 2015

7. Maurer CJ, Joachimski F, Berlis A: Two in one: endovascular treatment of acute tandem occlusions in the anterior circulation. Clin Neuroradiol 25:397-402, 2015

8. NINDS rt-PA Stroke Study Group: Tissue plasminogen activator for acute ischemic stroke. N Engl J Med 333:15811587,1995

9. Novakovic RL, Toth G, Narayanan S, Zaidat OO: Retrievable stents, "stentrievers," for endovascular acute ischemic stroke therapy. Neurology 79 (13 Suppl 1):S148-S157, 2012

10. Orrison WW Jr, Snyder KV, Hopkins LN, Roach CJ, Ringdahl EN, Nazir R, et al: Whole-brain dynamic CT angiography and perfusion imaging. Clin Radiol 66:566-574, 2011

11. Rahme R, Abruzzo TA, Ringer AJ: Acute ischemic stroke in the setting of cervical carotid occlusion: a proposed management strategy. World Neurosurg 76 (6 Suppl):S60-S65, 2011

12. Sivan-Hoffmann R, Gory B, Armoiry X, Goyal M, Riva R, Labeyrie PE, et al: Stent-retriever thrombectomy for acute anterior ischemic stroke with tandem occlusion: a systematic review and meta-analysis. Eur Radiol 27:247-254, 2017

13. Soize S, Kadziolka K, Estrade L, Serre I, Barbe C, Pierot L: Outcome after mechanical thrombectomy using a stent retriever under conscious sedation: comparison between tandem and single occlusion of the anterior circulation. J Neuroradiol 41:136-142, 2014

14. Steglich-Arnholm H, Holtmannspötter M, Kondziella D, Wagner A, Stavngaard T, Cronqvist ME, et al: Thrombectomy assisted by carotid stenting in acute ischemic stroke management: benefits and harms. J Neurol 262:2668-2675, 2015

15. Turk AS, Frei D, Fiorella D, Mocco J, Baxter B, Siddiqui A, et al: ADAPT FAST study: a direct aspiration first pass technique for acute stroke thrombectomy. J Neurointerv Surg 6:260-264, 2014

16. Villwock MR, Padalino DJ, Deshaies EM: Carotid artery stenosis with acute ischemic stroke: stenting versus angioplasty. J Vasc Interv Neurol 8:11-16, 2015

17. Widimský P, Koznar B, Abelson M, Blaško P, Lanzer P, Mazighi M, et al: Stent or balloon: How to treat proximal internal carotid artery occlusion in the acute phase of ischemic stroke? Results of a short survey. Cor Vasa 58:e204-e206, 2016

\section{Disclosures}

Dr. Levy has shareholder/ownership interests in Intratech Medical Ltd., Blockade Medical LLC, and NeXtGen Biologics. He is a principal investigator for Covidien US SWIFT PRIME Trials and receives honoraria for training and lectures from Covidien. Dr. Levy serves as a consultant for Pulsar and for Blockade Medical. He is a member of the Advisory Boards of Stryker, NeXtGen Biologics, and MEDX. He receives financial support from Abbott 
for carotid artery training sessions. Dr. Levy also renders medical/ legal opinion as an expert witness. Dr. Siddiqui has ownership in Buffalo Technology Partners Inc., Cardinal, International Medical Distribution Partners, Medina Medical Systems, Neuro Technology Investors, StimSox, and Valor Medical. He is a consultant for Amnis Therapeutics Ltd., Cerebrotech Medical Systems Inc., CereVasc LLC, Codman, Corindus Inc., Covidien (acquired by Medtronic), GuidePoint Global Consulting, Lazarus (acquired by Medtronic), Medina Medical (acquired by Medtronic), Medtronic, MicroVention, Neuravi, Penumbra, Pulsar Vascular, Rapid Medical, Rebound Medical, Reverse Medical (acquired by Medtronic), Silk Road Medical Inc., Stryker, The Stroke Project Inc., Three Rivers Medical Inc., and W.L. Gore \& Associates. Dr. Siddiqui is a principal investigator/national steering committee member of the Covidien SWIFT PRIME Trial, LARGE Trial, Medtronic SWIFT DIRECT, MicroVention CONFIDENCE Study, MicroVention
FRED Trial, Penumbra 3D Separator Trial, Penumbra COMPASS Trial, Penumbra INVEST Trial, and POSITIVE Trial. He is a board member of the Intersocietal Accreditation Committee. Dr. Snyder is a Speaker's Bureau member for Toshiba and Jacobs Institute.

\section{Author Contributions}

Conception and design: all authors. Acquisition of data: all authors. Analysis and interpretation of data: all authors. Drafting the article: Rangel-Castilla, Rajah. Critically revising the article: all authors. Reviewed submitted version of manuscript: all authors.

\section{Correspondence}

Adnan H. Siddiqui, Department of Neurosurgery, University at Buffalo Neurosurgery, 100 High St., Ste. B4, Buffalo, NY 14203. email: asiddiqui@ubns.com. 